\title{
A Spatial Model to Describe the Dengue Propagation $^{1}$
}

\author{
N.A. MAIDANA 2 , H.M. YANG ${ }^{3}$, Departamento de Matemática Aplicada, IMECC, \\ UNICAMP, Cx.P. 6065, 13083-970 Campinas, SP, Brazil.
}

\begin{abstract}
Dengue is a human disease transmitted by the mosquito Aedes aegypti. A dengue epidemic could start when infectious individuals (humans or mosquitoes) appear and could propagate in areas previously colonized by the mosquitoes. In this work, we propose a model to study the dengue propagation using a system of partial differential reaction-diffusion equations. The human and mosquito populations are considered, with their respective subclasses of infected and non infected populations. We assume that the diffusion occurs only in the winged form, and the human population is considered constant. The cross-infection is modeled by the mass action incidence law. A threshold value, as a function of the model's parameters, is obtained, which determines the endemic level of the disease. Assuming that an area was previously colonized by the mosquitoes, the spread velocity of the disease propagation is determined as a function of the model's parameters. The traveling waves solutions of the system of partial differential equations are considered to determine the spread velocity of the front wave.
\end{abstract}

\section{Introduction}

The dengue virus is an arbovirus transmitted by arthropod of the genus Aedes. The cosmo-tropical mosquito, Aedes aegypti (Linn. Diptera: Culicidae), serves as the most important domestic vector of dengue and urban yellow fever. The dengue virus is prevalent in different parts of the world presenting only one epidemiological cycle (urban) that comprises the human (host) and the mosquito Aedes aegypti (vector). As a result of being pathogenic for humans and capable of transmission in heavily populated areas, dengue virus can cause widespread and serious epidemics, appearing as a major public health problems in many tropical and subtropical regions of the world.

With respect to the disease, briefly, there are 4 different serotypes of dengue virus presenting low cross immunity among them, which can result in secondary infections after an infection with one serotype had been occurred. It is known that an individual infected with one serotype can be infected with other serotype six months later after the first exposure, but there is not evidence of re-infection with the same

\footnotetext{
${ }^{1}$ Apoio financeiro FAPESP (Bolsa PD e Projeto Temático)

${ }^{2}$ Fellowship FAPESP (nmaidana@ime.unicamp.br)

${ }^{3}$ Fellowship CNPq (hyunyang@ime.unicamp.br)
} 
serotype. Dengue viruses of all four serotypes cause three distinct syndromes: classic dengue fever, dengue hemorrhagic fever and dengue shock syndrome. Although caused by the same viruses, dengue and dengue hemorrhagic fever are pathogenetic, clinically and epidemiologically distinct.

In Brazil dengue disease is actually one of the main public health challenges showing an increasing in number of cases and in geographic distribution through time. For instance, in 1998, 537.507 cases of dengue were registered in 24 States, among them 98 cases of hemorrhagic dengue occurred in 9 States. In the same year, in the São Paulo State, 10.629 cases of dengue disease occurred in 102 municipalities, overcoming increased efforts spent by the public health authorities in the control of the vector $A$. aegypti. The resurgence of dengue transmission in Brazil is due to the socio-economic conditions getting worse, the change in the climatic conditions due to the global warming up, the "El Niño" phenomenon, discontinuous sanitary activities and others.

In this paper we deal with the invasion and colonization of mosquitoes $A$. aegypti followed by the establishment of dengue epidemics. In section 2 we develop a model for dengue transmission, which is analyzed in section 3 to obtain traveling waves solutions. Finally in section 4 we provided conclusion.

\section{Model for the Disease Dynamic}

The dengue disease is a vector-borne viral infection transmitted among humans by mosquito's bite during the blood meal. We propose a model taking into account the human and the mosquito populations. In the human population we consider the subpopulations of susceptible, infected and removed individuals. The spatial density at time $t$ is denoted by $\bar{H}(x, t), \bar{I}(x, t)$ and $\bar{R}(x, t)$, respectively. To the mosquito population we consider the winged and an aquatic subpopulations, which includes eggs, larvae and pupae, denoted by $\bar{M}(x, t)$ and $\bar{A}(x, t)$, respectively [5]. The human population is under a constant per-capita mortality rate $\overline{\mu_{H}}$.

With respect to the winged form, the non infected and the infected classes are considered, which are designed by $\bar{M}_{S}(x, t)$ and $\bar{M}_{I}(x, t)$, respectively. The total population in the winged form is $\bar{M}(x, t)=\bar{M}_{S}(x, t)+\bar{M}_{I}(x, t)$. In the mosquito population, the intrinsic oviposition rate is denoted by $r$, with the aquatic form being constrained by the carrying capacity $k_{2}$, and per-capita mortality rate $\mu_{2}$. The per-capita rate of maturation of the aquatic form into the winged is denoted by $\gamma$, with the adult mosquito being saturated by the carrying capacity $k_{1}$. The per-capita mortality rate of the winged is $\mu_{1}$.

With respect to the transmission of the dengue infection, we assume the mass action law with two constant transmission coefficients. One is the transmission

coefficient $\overline{\beta_{1}}$, which measures the rate of effective contact between non infected mosquitoes with infected human. The other is $\overline{\beta_{2}}$, which measures the rate of effective contact between non infected humans with infected mosquitoes. Considering the natural history of infection, in the human population the removal rate is denoted by $\bar{\sigma}$, which transfers infectious individuals to removed class. We are not considering immunity among mosquitoes. 
Finally, the geographical dispersal of $A$. aegypti among spatially fixed humans is assumed. Both infected and non infected classes of mosquitoes are under diffusion by wings and advection by winds, described by the diffusion parameter $\bar{D}$ and advection $\bar{\nu}$.

The model, which governs the spatial and temporal evolution of the disease, is the following

$$
\begin{aligned}
\frac{\partial \overline{M_{S}}}{\partial t} & =\bar{D} \frac{\partial^{2} \overline{M_{S}}}{\partial x^{2}}-\bar{\nu} \frac{\partial \overline{M_{S}}}{\partial x}+\bar{\gamma} \bar{A}\left(1-\frac{\bar{M}}{k_{1}}\right)-\overline{\mu_{1}} \overline{M_{S}}-\overline{\beta_{1}} \overline{M_{S}} \bar{I} \\
\frac{\partial \overline{M_{I}}}{\partial t} & =\bar{D} \frac{\partial^{2} \overline{M_{I}}}{\partial x^{2}}-\bar{\nu} \frac{\partial \overline{M_{I}}}{\partial x}-\overline{\mu_{1}} \overline{M_{I}}+\overline{\beta_{1}} \overline{M_{S}} \bar{I} \\
\frac{\partial \bar{A}}{\partial t} & =r\left(1-\frac{\bar{A}}{k_{2}}\right) \bar{M}-\overline{\mu_{2}} \bar{A}-\bar{\gamma} \bar{A} \\
\frac{\partial \bar{H}}{\partial t} & =\overline{\mu_{H}} \bar{N}-\overline{\mu_{H}} \bar{H}-\overline{\beta_{2}} \bar{H} \overline{M_{I}} \\
\frac{\partial \bar{I}}{\partial t} & =\overline{\beta_{2}} \bar{H} \overline{M_{I}}-\bar{\sigma}-\overline{\bar{\mu}} \bar{I} \\
\frac{\partial \bar{R}}{\partial t} & =\bar{\sigma} \bar{I}-\overline{\mu_{H}} \bar{R} .
\end{aligned}
$$

The system of equations (2.1)-(2.6) is manipulated disregarding the dengue transmission. Summing the last three equations of the system, we obtain the density of the human population $N, N=H+I+R$, given by

$$
\frac{\partial \bar{N}}{\partial t}=\frac{\partial \bar{H}}{\partial t}+\frac{\partial \bar{I}}{\partial t}+\frac{\partial \bar{R}}{\partial t}=0,
$$

because we assumed that the human population is constant. Summing first two equations of the system, with $\bar{M}(x, t)=\bar{M}_{S}(x, t)+\bar{M}_{I}(x, t)$, we have the equations for the dispersal of mosquito population

$$
\begin{aligned}
\frac{\partial \bar{M}}{\partial t} & =\bar{D} \frac{\partial^{2} \bar{M}}{\partial x^{2}}-\bar{\nu} \frac{\partial \bar{M}}{\partial x}+\bar{\gamma} \bar{A}\left(1-\frac{\bar{M}}{k_{1}}\right)-\overline{\mu_{1}} \bar{M} \\
\frac{\partial \bar{A}}{\partial t} & =r\left(1-\frac{\bar{A}}{k_{2}}\right) \bar{M}-\overline{\mu_{2}} \bar{A}-\bar{\gamma} \bar{A} .
\end{aligned}
$$

This is the system of equations analyzed in [5] for the A. aegypti dispersal dynamics in the absence of disease.

We now introduce the non dimensional parameters to the system of equations (2.1)-(2.6). The mosquito population, in their winged and aquatic phases, are scaled by its carrying capacity; the time is scaled with respect to the ovipotition rate $r$ and for the spatial scaling, the quotient between the mosquito diffusion and the 
ovipotition rate according to $\sqrt{\frac{D}{r}}$. The dimensionless parameters are

$$
\begin{aligned}
M_{S} & =\frac{\overline{M_{S}}}{k_{1}}, \quad M_{I}=\frac{\overline{M_{I}}}{k_{1}}, \quad A=\frac{\bar{A}}{k_{2}}, \quad H=\frac{\bar{H}}{\overline{H_{0}}}, \quad I=\frac{\bar{I}}{\overline{H_{0}}}, \quad R=\frac{\bar{R}}{\overline{H_{0}}} \\
k & =\frac{k_{1}}{k_{2}}, \quad \nu=\frac{\bar{\nu}}{r}\left(\frac{r}{D}\right)^{1 / 2}, \quad \gamma=\frac{\gamma}{r}, \quad \mu_{1}=\frac{\overline{\mu_{1}}}{r}, \quad \mu_{2}=\frac{\overline{\mu_{2}}}{r} \\
\beta_{1} & =\frac{\overline{\beta_{1}} \overline{H_{0}}}{r}, \quad \beta_{2}=\frac{\overline{\beta_{2}} k_{1}}{r}, \quad \mu_{H}=\frac{\overline{\mu_{H}}}{r}, \quad \sigma=\frac{\bar{\sigma}}{r} .
\end{aligned}
$$

Therefore, the dimensionless model is

$$
\begin{aligned}
\frac{\partial M_{S}}{\partial t} & =\frac{\partial^{2} M_{S}}{\partial x^{2}}-\nu \frac{\partial M_{S}}{\partial x}+\frac{\gamma}{k} A(1-M)-\mu_{1} M_{S}-\beta_{1} M_{S} I \\
\frac{\partial M_{I}}{\partial t} & =\frac{\partial^{2} M_{I}}{\partial x^{2}}-\nu \frac{\partial M_{I}}{\partial x}-\mu_{1} M_{I}+\beta_{1} M_{S} I \\
\frac{\partial A}{\partial t} & =k(1-A) M-\mu_{2} A-\gamma A \\
\frac{\partial H}{\partial t} & =\mu_{H} N-\mu_{H} H-\beta_{2} H M_{I} \\
\frac{\partial I}{\partial t} & =\beta_{2} H M_{I}-\sigma I-\mu_{H} I \\
\frac{\partial R}{\partial t} & =\sigma I-\mu_{H} R .
\end{aligned}
$$

With respect to the dimensionless model, equations (2.7)-(2.12), there are three steady states. The steady state with only the human population is given by

$$
E_{0}=\left(M_{S}^{0}, M_{I}^{0}, A^{0}, H^{0}, I^{0}, R^{0}\right)=(0,0,0,1,0,0),
$$

which is the areas free of mosquitoes where traveling wave front of mosquitoes can colonized these areas [5].

The second equilibrium is the case when the mosquitoes are well established in the region

$$
E_{1}=\left(M_{S}^{1}, M_{I}^{1}, A^{1}, H^{1}, I^{1}, R^{1}\right)=\left(m^{*}, 0, a^{*}, 1,0,0\right),
$$

where

$$
a^{*}=\frac{k \gamma-\mu_{1} k\left(\mu_{2}+\gamma\right)}{k \gamma+\gamma\left(\mu_{2}+\gamma\right)} \quad \text { and } \quad m^{*}=\frac{\gamma-\mu_{1}\left(\mu_{2}+\gamma\right)}{\mu_{1} k+\gamma} .
$$

The biological condition, for the existence of the mosquito population, implies that

$$
\gamma-\mu_{1}\left(\mu_{2}+\gamma\right)>0
$$

The third steady state, which corresponds to the disease at an endemic level, is 
given by

$$
\begin{aligned}
E^{*} & =\left(M_{S}^{*}, M_{I}^{*}, A^{*}, H^{*}, I^{*}, R^{*}\right), \\
M_{S}^{*} & =m^{*}-M_{I}^{*}, \quad M_{I}^{*}=\frac{\left(1-H^{*}\right) \mu_{H}}{\beta_{2} H^{*}}, \\
A^{*} & =a^{*}, \\
H^{*} & =\frac{\beta_{1} \mu_{H}+\mu_{1}\left(\mu_{H}+\sigma\right)}{\beta_{1} \mu_{H}+\beta_{1} \beta_{2} m^{*}}, \\
I^{*} & =\frac{\left(1-H^{*}\right) \mu_{H}}{\mu_{H}+\sigma}, \\
R^{*} & =1-H^{*}-I^{*} .
\end{aligned}
$$

For the disease being prevalent is necessary that $H^{*}<1$. From this condition we obtain the basic reproducibility number

$$
R_{0}=\frac{\beta_{1} \beta_{2} m^{*}}{\mu_{1}\left(\mu_{H}+\sigma\right)},
$$

where if $R_{0}>1$ the epidemic occurs, since satisfies $H^{*}<1$. The basic reproducibility number is the threshold value, which determines the endemic level of the disease. This is important for control strategies.

Table 1: Parameters for the A. aegypti.

\begin{tabular}{lcl}
\hline Parameter & Symbol & Value \\
\hline Diffusion coefficient & $\bar{D}$ & $1,25 \times 10^{-2} \mathrm{~km}^{2} /$ days \\
Advection coefficient & $\bar{\nu}$ & $5 \times 10^{-2} \mathrm{~km} /$ days \\
Maturation rate & $\bar{\gamma}^{-1}$ & 5 days \\
Oviposition rate & $\bar{r}$ & 30 days \\
Carrying capacity - winged form & $k_{1}$ & 25 individuals $/ \mathrm{km}^{2}$ \\
Carrying capacity - aquatic form & $k_{2}$ & 100 individuals $/ \mathrm{km}^{2}$ \\
Mortality rate in winged phase & $\bar{\mu}_{1}^{-1}$ & 25 days \\
Mortality rate in aquatic phase & $\bar{\mu}_{2}^{-1}$ & 100 days \\
\hline
\end{tabular}

The values of the model's parameters are obtained from literature. The data in table 1 are those encountred in [5]. With respect to data in Table 2, the mortality correponds to developing countries, and the infectious period of dengue is the range 5-10 days [6]. The number of humans correspond to the population of São Paulo state per $\mathrm{km}^{2}$ (considering the total population divided by the state area). Finally, the contact rates are chosen arbitrarity.

For the dimensionless parameters, corresponding to those listed in Tables 1 and 2 , the basic reproducibility number is $R_{0}=6.57$. In this case the epidemic occurs, and it could propagate in the region previously free of disease.

We study the existence of the traveling waves solutions connecting the steady states $E_{1}$ and $E_{*}$, i.e., when there are mosquitoes in the region, and the disease begins to propagate. 
Table 2: Parameters for the disease transmission.

\begin{tabular}{lcl}
\hline Parameter & Symbol & Value \\
\hline $\begin{array}{l}\text { Contact rate - infection among } \\
\text { mosquitoes }\end{array}$ & $\overline{\beta_{1}}$ & 0.015 \\
$\begin{array}{l}\text { Contact rate - infection among } \\
\text { humans }\end{array}$ & $\overline{\beta_{2}}$ & 0.0007 \\
$\begin{array}{l}\text { Life expectancy } \\
\text { Infected period }\end{array}$ & ${\overline{\mu_{H}}}^{-1}$ & 60 years \\
Intial number of humans & $\bar{\sigma}^{-1}$ & 7 days \\
\hline
\end{tabular}

\section{Dengue Propagation: traveling waves solutions}

Considering that the mosquito population is well established in a region, we analyze the propagation of the dengue disease when small number of infective individuals are introduced in this community. We seek for the existence of traveling waves solutions which must connect $E_{1}$ and $E^{*}$. We must determine the minimum speed of the traveling waves, because it is stable and represents the observable trajectory of the dynamics system [4] [7].

The traveling waves solutions, when there exists, must be represented by

$\left(m_{s}(x, t), m_{i}(x, t), a(x, t), h(x, t), i(x, t), r(x, t)\right)=\left(m_{s}(z), m_{i}(z), a(z), h(z), i(z), r(z)\right)$,

where $z=x+c t$, and $c$ is the constant velocity [1]. Applying this change of variable to the system of equations (2.7)-(2.12), we obtain the corresponding dimensionless system of the first order ordinary differential equations given by

$$
\begin{aligned}
\frac{d m_{s}}{d z} & =u, \\
\frac{d u}{d z} & =(c+\nu) u-\frac{\gamma}{k}(1-m) a+\mu_{1} m_{s}+\beta_{1} m_{s} i \\
\frac{d m_{i}}{d z} & =v \\
\frac{d v}{d z} & =(c+\nu) v+\mu_{1} m_{i}-\beta_{1} m_{s} I \\
\frac{d a}{d z} & =(1 / c)\left(k(1-a) m-\mu_{2} a-\gamma a\right) \\
\frac{d h}{d z} & =(1 / c)\left(\mu_{H} n-\mu_{H} h-\beta_{2} h m_{i}\right) \\
\frac{d i}{d z} & =(1 / c)\left(\beta_{2} h m_{i}-\sigma i-\mu_{h} i\right) \\
\frac{d r}{d z} & =(1 / c)\left(\sigma i-\mu_{H} r\right) .
\end{aligned}
$$

Let us analyze the stability of the equilibrium point $E_{1}$ with respect to the linear system of equations (3.1)-(3.8). The eigenvalues are

$$
\lambda_{1}=0, \quad \lambda_{2}=-\frac{\mu_{H}}{c},
$$


and the roots of the two polynomials of third degree

$$
\begin{aligned}
P_{1}(\lambda)= & \lambda^{3}+\left(-(c+\nu)+\frac{\left(\mu_{H}+\sigma\right)}{c}\right) \lambda^{2}-\left(\mu_{1}+(c+\nu) \frac{\left(\mu_{H}+\sigma\right)}{c}\right) \lambda \\
& +\frac{\beta_{1} \beta_{2} m^{*}-\mu_{1}\left(\mu_{H}+c\right)}{c} \\
P_{2}(\lambda)= & \lambda^{3}+\left(-(c+\nu)+\frac{\gamma+\mu_{2}+k m^{*}}{c}\right) \lambda^{2} \\
& +\left(-(c+\nu) \frac{\left(\gamma+k m^{*}+\mu_{2}\right)}{c}-\left(\frac{a^{*} \gamma+k \mu_{1}}{k}\right)\right) \lambda-\frac{\gamma\left(1-\mu_{1}\right)-\mu_{2} \mu_{1}}{c} .
\end{aligned}
$$

Since the variables are population, we constrain to the dynamical system the fact that the solution must not oscillate around the origin, i.e., the eigenvalues must assume real values. The polynomial $P_{2}$ is structured in terms of the parameters regarded to the mosquito population and does not comprise neither human population's parameters nor infection's parameters. Let us apply the Cardan formula [3] and write the discriminant of $P_{2}$ as a new polynomial in $c$. The discriminant has the form

$$
\operatorname{dis}(c)=\operatorname{pol}(c) / c^{4},
$$

where $\operatorname{pol}(c)=\left(a_{6} c^{6}+a_{4} c^{4}+a_{2} c^{2}+a_{0}\right)$ and the coefficients $a_{i}$, for $i=0,2,4,6$, depend on the parameters $\gamma, \mu_{1}, \mu_{2}$ and $k$.

For values of the parameters of $A$. aegypti given in Table 1 , setting $\nu=0$, the discriminant is less than zero, and the polynomial has only real roots (see Figure $1)$. In this case, the value of $\gamma$ is 0.0066 . When this value decreases below to the critical value $\gamma_{c}=0.00000044481$, in which case the mosquito population does not exist, we have $a^{*}=0$ and $m^{*}=0$, and the maximum of the polynomial $\operatorname{pol}(c)$ is increased, but never assumes valued less than zero. At this critical situation the maximum of $\operatorname{pol}(c)$ is zero in the origin (see Figure 1). This is a consequence of the condition (2.13) being equal to zero and the independent coefficient,

$$
a_{0}=\frac{\left(\gamma-\mu_{1}\left(\mu_{2}+\gamma\right)\right) 4 \gamma^{3}(\gamma+k+\mu)^{3}}{27\left(\gamma+k \mu_{1}\right)^{3}}
$$

is also zero.

Finally, the minimum traveling waves velocity is obtained from the polynomial $P_{1}(\lambda)$, denoted $c_{m i n}$. The condition $R_{0}>1$ implies that $P_{1}(0)>0$. Besides, we must have

$$
\lim _{\lambda \rightarrow \pm \infty} P_{1}(\lambda)= \pm \infty,\left.\quad \frac{d P_{1}(\lambda)}{d \lambda}\right|_{\lambda=0}<0
$$

in order to $P_{1}(\lambda)$ has always negative real root. Then, the double positive real roots determine $c_{\text {min }}$ (see Figure 2). For all $c<c_{\text {min }}$ there are complex solutions for $P_{1}(\lambda)$, and for $c \geq c_{m i n}$, all the roots are real. Imposing that $c_{m i n}$ must be double real roots, we obtain equation to determine $c_{\min }$ as a function of the parameters. The equation is the following

$$
\lambda^{3}+\left(-(c+\nu)+\frac{\left(\mu_{H}+\sigma\right)}{c}\right) \lambda^{2}-\left(\mu_{1}+(c+\nu) \frac{\left(\mu_{H}+\sigma\right)}{c}\right) \lambda+\frac{\beta_{1} \beta_{2} m^{*}-\mu_{1}\left(\mu_{H}+c\right)}{c}=0
$$



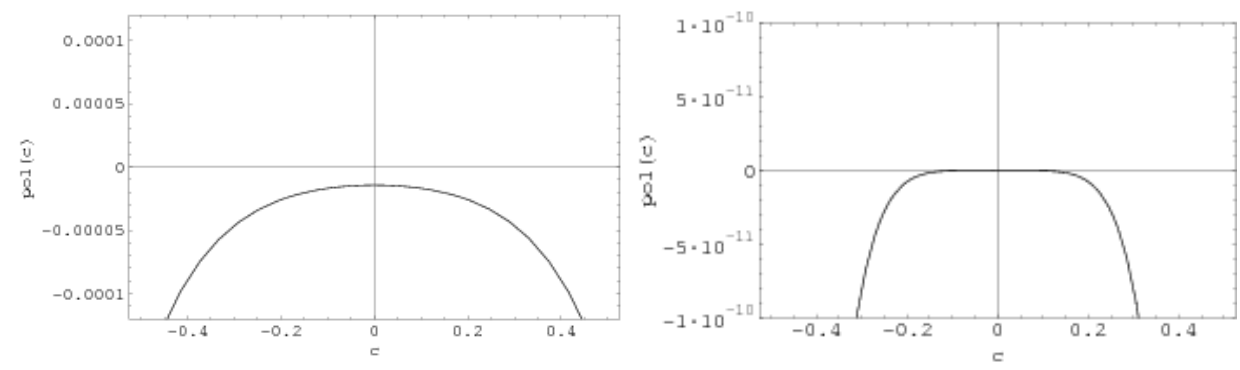

Figure 1: Left: Graph of the polynomial $\operatorname{pol}(c)$ for the parameters listed in Table 1. Right: Graph of the polynomial pol(c) for the parameters listed in Table 1, setting $\gamma_{c}=0.00000044481$, driving to $a^{*}=0$ and $m^{*}=0$. The maximum is reached in the origin at this critical value.

or

$\lambda=\frac{1}{3}\left\{-\left(-(c+\nu)+\frac{\left(\mu_{H}+\sigma\right)}{c}\right) \pm \sqrt{\left(-(c+\nu)+\frac{\left(\mu_{H}+\sigma\right)}{c}\right)^{2}+3\left(\mu_{1}+(c+\nu) \frac{\left(\mu_{H}+\sigma\right)}{c}\right)}\right\}$

The dimensionless speed, for the parameters listed in Tables 1 and 2, setting $\nu=0$, is $c_{\min }=0.1009$. In the original parameters this speed of propagation of the dengue disease is: $0.06 \mathrm{~km} /$ days, which did not consider the advection movement. In Figure 3, the traveling waves are showed for the sub-populations of infected and non infected humans, and for infected and non infected mosquitoes. A cyclic front wave of the disease can be observed.

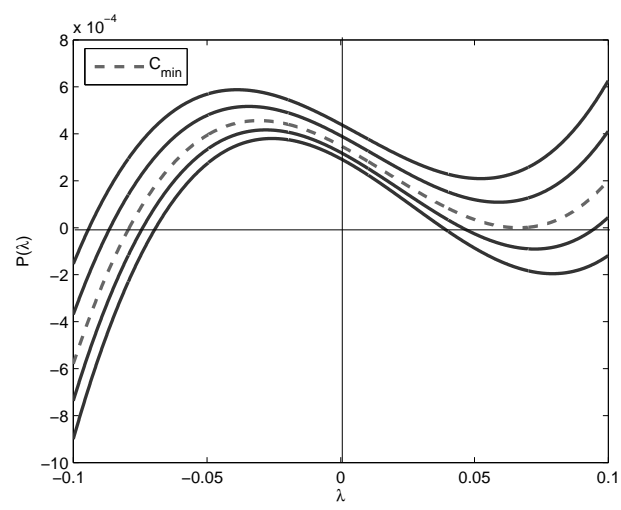

Figure 2: Graph of the polynomial $P_{1}(\lambda)$ for the parameters listed in Table 1, setting $\nu=0$, and Table2. The curves correspond to the values $c=0.12, c=0.11$, with three real roots, $c_{\min }=0.1009$ with a double root, and $c=0.09$ and $c=0.08$ with only one real root. 


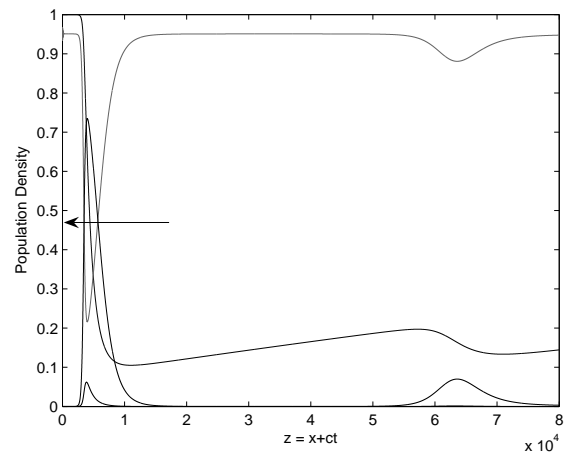

Figure 3: Traveling waves for the parameters listed in Tables 1 and 2, setting $\nu=0$, for infected and non infected humans, and infected and non infected mosquitoes. A front wave of the disease can be observed, followed by a second front.

\section{Conclusion}

In this work we first determined the basic reproducibility number, which determines the endemic level of the disease. Then the minimum speed of the traveling waves was determinated, which describes the propagation of dengue in an area previously colonized by the mosquito $A$. aegypti. The equation with respect to the minimum traveling waves velocity was determined, as function of the model's parameters. This permits to evaluate control strategies in order to attain the eradication of dengue transmission by decreasing the speed of propagation up to zero, which is left to a future work.

In this work, the advection were not considered $(\nu=0)$. The advection, in a preferential direction, increases the wave velocity and this phenomenon will be considered in a future work.

Resumo. Dengue é uma doença entre humanos transmitida por mosquito Aedes aegypti. Uma epidemia de dengue pode ser deflagrada quando indivíduos infecciosos (humanos ou mosquitos) surgem e a doença propaga-se em áreas previamente colonizadas por mosquitos. Nesse trabalho, propomos um modelo matemático para analisar a propagação de dengue usando um sistema de equações diferenciais parciais de reação-advecção. As populações humana e de mosquitos são consideradas, com suas respectivas sub-populações de infectados e não infectados. Assumimos que a difusão só ocorre na forma alada dos mosquitos, e a população humana é constante. As infecções cruzadas entre as duas populações são modeladas assumindo a lei da ação das massas para incidências. Do modelo obtém-se um valor limiar, em função dos parâmetros do modelo, que determina o nível de endemicidade da infecção. Assumindo que uma área fora previamente colonizada por mosquitos, a velocidade de espalhamento da doença é determinada em função dos parâmetros do modelo. A solução de ondas viajantes para o sistema de equações parciais fornece a velocidade de frente de onda. 


\section{References}

[1] J.D. Murray, "Mathematical Biology", Springer, Berlin, 2002.

[2] J.D. Murray, F.R.S. Stanley, D.L. Brown, On the spatial spread of rabies amog foxes, Proc. R. Soc. Lond., B229 (1986), 111-150.

[3] J. Rotman, "Galois Theory", New York Springer, 1990.

[4] B. Sandstede, Stability of traveling waves, in "Handbook of Dynamical System II", (B. Fiedler, ed.) pp. 983-1059, Elsevier, Amsterdam, 2002.

[5] L.T. Takahashi, N.A. Maidana, W.C. Ferreira Jr., P. Pulino, H.M. Yang, Mathematical models for the Aedes aegypti dispersal dynamics: traveling waves by wing and wind, Bulletin of Mathematical Biology, 67 (2005), 509-528.

[6] R. Veronesi, "Doenças Infecciosas e Parasitárias", Guanabara Koogan S.A., 1991.

[7] A.I. Volpert, V.A. Volpert, "Traveling Waves Solutions of Parabolic System", American Mathematical Society, Providence, RI, 1994. 